\title{
Prediction of functional loss in emergency surgery is possible with a simple frailty screening tool
}

Davide Zattoni ${ }^{1,2^{*}} \mathbb{D}$, Isacco Montroni ${ }^{2}$, Nicole Marie Saur ${ }^{3}$, Anna Garutti ${ }^{4}$, Maria Letizia Bacchi Reggiani ${ }^{5}$, Federico Ghignone ${ }^{2}$, Giovanni Taffurelli ${ }^{2}$ and Giampaolo Ugolini ${ }^{1,2}$

\begin{abstract}
Background: Senior adults fear postoperative loss of independence the most, and this might represent an additional burden for families and society. The number of geriatric patients admitted to the emergency room requiring an urgent surgical treatment is rising, and the presence of frailty is the main risk factor for postoperative morbidity and functional decline. Frailty assessment in the busy emergency setting is challenging. The aim of this study is to verify the effectiveness of a very simple five-item frailty screening tool, the Flemish version of the Triage Risk Screening Tool (fTRST), in predicting functional loss after emergency surgery among senior adults who were found to be independent before surgery.

Methods: All consecutive individuals aged 70 years and older who were independent (activity of daily living (ADL) score $\geq 5$ ) and were admitted to the emergency surgery unit with an urgent need for abdominal surgery between December 2015 and May 2016 were prospectively included in the study. On admission, individuals were screened using the fTRST and additional metrics such as the age-adjusted Charlson Comorbidity Index (CACl) and the ASA score. Thirty- and 90-day complications and postoperative decline in the ADL score where recorded. Regression analysis was performed to identify preoperative predictors of functional loss.

(Continued on next page)
\end{abstract}

\footnotetext{
* Correspondence: davide.zattoni@auslromagna.it; davidezattoni@gmail.com

Suggested reviewers

Siri Rostoft, Geriatrics, University of Oslo, srostoft@gmail.com

Armin Shahrokni, Oncologist and geriatrics, Memorial Sloan Kettering Cancer

Center, shahroka@mskcc.org

'Department of General Surgery, Policlinico S. Orsola-Malpighi, 40138

Bologna, Italy

${ }^{2}$ Department of General Surgery, Ospedale per gli Infermi, Viale Stadone, 9, 48018 Faenza, Italy

Full list of author information is available at the end of the article
}

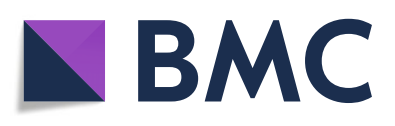

(c) The Author(s). 2021 Open Access This article is licensed under a Creative Commons Attribution 4.0 International License, which permits use, sharing, adaptation, distribution and reproduction in any medium or format, as long as you give appropriate credit to the original author(s) and the source, provide a link to the Creative Commons licence, and indicate if changes were made. The images or other third party material in this article are included in the article's Creative Commons licence, unless indicated otherwise in a credit line to the material. If material is not included in the article's Creative Commons licence and your intended use is not permitted by statutory regulation or exceeds the permitted use, you will need to obtain permission directly from the copyright holder. To view a copy of this licence, visit http://creativecommons.org/licenses/by/4.0/ The Creative Commons Public Domain Dedication waiver (http://creativecommons.org/publicdomain/zero/1.0/) applies to the data made available in this article, unless otherwise stated in a credit line to the data. 
(Continued from previous page)

Results: Seventy-eight patients entered the study. Thirty-day mortality rate was $12.8 \%$ (10/78), and the 90-day overall mortality was $15.4 \%$ (12/78). One in every four patients (17/68) experienced a significant functional loss at 30-day follow-up. At 90-day follow-up, only 3/17 patients recovered, 2 patients died, and 12 remained permanently dependent. On the regression analysis, a statistically significant correlation with functional loss was found for fTRST, $\mathrm{CACl}$, and age $\geq 85$ years old both at 30 and 90 days after surgery. fTRST $\geq 2$ showed the highest effectiveness in predicting functional loss at 90 days with AUC 72 and OR 6.93 (95\% Cl 1.71-28.05). The institutionalization rate with the need to discharge patients to a healthcare facility was 7.6\% (5/66); all of them had a fTRST $\geq 2$.

Conclusion: fTRST is an easy and effective tool to predict the risk of a postoperative functional decline and nursing home admission in the emergency setting.

Keywords: Functional outcome, Functional decline, Frailty assessment, Flemish version of Triage Risk Screening Tool, Emergency surgery

\section{Background}

An increased number of senior adults are admitted to the emergency department requiring urgent or emergent surgical care $[1,2]$. This population experiences poor surgical outcomes with high rates of postoperative complications, mortality, resource use, and a greater chance of being dependent at hospital discharge [3, 4]. Emergency surgery can also promote functional decline secondary to preoperative deconditioning and/or postoperative complications [5]. Losing preoperative abilities such as mental capacity, continence, mobility, or independence in daily activities is the most-feared postoperative event among geriatric patients [6] and could lead to sudden requirements for the assistance of a caregiver or discharge to a nursing facility [7]. Loss of independence impacts a patient's quality of life and represents an economic and social burden for families and society [4].

The most important risk factor for functional decline is frailty $[4,8,9]$. The surgeon has few instruments to predict a postoperative functional loss in the busy emergency setting where a frailty assessment in not available or reliable $[2,7,10]$. A short frailty screening tool validated in a previous study, the Flemish version of the Triage Risk Screening Tool (fTRST), is effective in predicting 30- and 90-day morbidity and mortality after emergency abdominal surgery among older patients $(\geq 70$ years) [10].

The aim of this study is to analyze if the fTRST can also predict loss of independence in a group of previously independent senior adults undergoing emergency abdominal surgery.

\section{Methods}

\section{Study population}

Between December 2015 and May 2016, patients 70 years old and older were prospectively enrolled before undergoing emergency abdominal surgery at a tertiary hospital. Patients with a preoperative ADL score of $6 / 6$ or $5 / 6$ at the preoperative baseline were considered functionally independent and included in the study. The study was conducted under the Institutional Review Board (214/2016/O/OssN). Informed consent was obtained from every patient, and a health care proxy was used in cases of dementia or altered mental status.

\section{Study protocol}

On admission, demographic data, activities of daily living (ADL), walking ability, Charlson Comorbidity Age Adjusted (CACI) score, the fTRST, and the American Society of Anesthesiology (ASA) score were recorded in a dedicated database.

The study-specific baseline assessment was performed by a senior general surgery resident (DZ) together with the resident on call who performed the standard admission history and physical exam, upon patient admission to the emergency surgery unit (ESU). In the case of emergent surgery, or if patients were incoherent, baseline assessment was obtained in the immediate postoperative period via interview of a family member.

Type and duration of surgery and postoperative morbidity and mortality were also recorded (DZ). Outcomes were re-assessed at 30 and 90 days after surgery. Followup consisted of an outpatient visit or a phone encounter depending on the patient's status (DZ). Caregivers were interviewed in cases where patients were unable to answer.

\section{Functional loss risk factors}

Preoperative frailty screening tools have been well established in the literature [10-16]. Thresholds have been reported as follows: fTRST score $\geq 2$, ASA score $\geq 4$, CACI $\geq 6$, major surgery, and age $\geq 85$ years old. The fTRST (Table 1) is based on five domains: presence of cognitive decline (2 points), living alone or caregiver not available or able (1 point), reduced mobility (necessity of a cane/walker or a caregiver's aid) or falls in the past 6 months (1 point), hospitalized in the past 3 months (1 point), and polypharmacy (the cutoff for polypharmacy 
Table 1 The Flemish version of the Triage Risk Screening Tool

\begin{tabular}{|c|c|c|}
\hline \multirow{2}{*}{$\begin{array}{l}\text { Items of "The Flemish version of the Triage Risk } \\
\text { Screening Tool" }\end{array}$} & \multicolumn{2}{|c|}{ Score } \\
\hline & Yes & No \\
\hline $\begin{array}{l}\text { 1. Presence of cognitive impairment (disorientation, } \\
\text { diagnosis of dementia, or delirium) }\end{array}$ & 2 & 0 \\
\hline 2. Lives alone or no caregiver available, willing, or able & 1 & 0 \\
\hline $\begin{array}{l}\text { 3. Difficulty with walking or transfers or fall(s) in the } \\
\text { past } 6 \text { months }\end{array}$ & 1 & 0 \\
\hline 4. Hospitalized in the last 3 months & 1 & 0 \\
\hline 5. Polypharmacy: $\geq 5$ medications & 1 & 0 \\
\hline
\end{tabular}

is established for this test when the patient takes $\geq 5$ different medications) (1 point). The fTRST cutoff was selected based on previous analysis [10], and it is in line with the cutoff chosen by Kenis et al. in the medical oncology setting [17]. Elevated CACI score was considered a predictor of morbidity and mortality $[12,13]$. Based on past works $[10,12]$, the cutoff was established as $\geq 6$. "Major surgery" was defined as a surgical intervention involving $\geq 1$ bowel resection or at least one anastomosis, gastric resection, splenectomy, or surgery performed for diffuse peritonitis. Bowel surgery as adhesiolysis or stoma creation without resection, appendectomies, laparoscopic cholecystectomies (without diffuse peritonitis), or hernia repair are considered intermediate-minor procedures. ASA score $\geq 4$ has been described as a significant risk factor for morbidity and mortality in the geriatric population undergoing emergency procedure $[18,19]$. As age is often a surrogate for frailty and since age has been included as a variable in past studies, it was included in this analysis, and people 85 and older were also considered at higher risk for postoperative complications [20].

\section{Outcome measures}

Patients were considered to be independent on the activities of daily living if ADL score was $6 / 6$ or $5 / 6$ on admission. A functional loss was established when a postoperative decline on ADL was observed 30 and 90 days after surgery from ADL 5-6 to ADL $\leq 4$.

All complications occurring during the hospital stay or within 90 days from discharge were recorded according to the Clavien-Dindo (CD) classification. $C D \geq 3$ was considered a major complication. Postoperative outcomes were recorded during the hospital stay by a senior resident (DZ). The 30- and 90-day follow-up was assessed via office visit or phone encounter with patients and/or caregivers.

Patients were considered to be institutionalized when a permanent transfer to a nursing facility occurred for patients previously living at home.

\section{Statistical analysis}

Statistical analyses were performed using Stata/SE 14.1 for Windows; continuous variables were expressed as mean \pm standard deviation or median and interquartile range; categorical data were expressed as numbers (percentages). For group comparisons of categorical and continuous variables, chi-square test, Fisher's exact test, Student's $t$-test, or Mann-Whitney test was used, as appropriate.

Logistic regression analyses were performed in order to evaluate pre- and intraoperative variables as risk factors (or predictors) of 30-day and 90-day "significant" functional loss $(\Delta \geq 2$ points on ADL score). Included risk variables were fTRST score $\geq 2$, ASA score $\geq 4$, CACI $\geq$ 6 , major surgery intervention, and age $\geq 85$ years old.

The model discrimination and calibration were reported together with AIC (Akaike information criterion) and BIC (Bayesian information criterion) measures for comparing maximum likelihood models. Model discrimination was assessed calculating the area under the receiver operating characteristic (ROC) curve (AUC), whereas model calibration has been determined by Hosmer-Lemeshow (H-L) technique. All $p$ values refer to two-tailed tests of significance. $p<0.05$ was considered significant. Given two models fit on the same data, the model with the smaller value of the information criterion is considered to be better.

\section{Results}

\section{Patient characteristics}

Between December 2015 and May 2016, 110 consecutive patients 70 years old and older underwent an abdominal urgent intervention under general anesthesia. Population diagram is reported on Fig. 1. Seventy-eight independent individuals on ADL (78/110; 70.9\%) were included in the analysis.

No eligible patient refused to participate in the study or was lost at follow-up. Twenty-seven percent of the population included in the study analysis was $\geq 85$ years of age or older (16 patients 70-74 years, 25 patients 7579 years, 16 patients $80-84$ years, 15 patients $85-89$ years, 6 patients $\geq 90$ years old). Median age was 78 years (range 70-96). Most of the patients came to the emergency room from home (65.4\%), 26 patients were transferred from other units (33.3\%), and only 1 patient lived in a nursing facility before admission.

Demographic data and patients' conditions on admission are included in Table 2. Among patients with ADL $\geq 6,85 \%$ had a score of $6 / 6$ while 12 patients had a score of 5 (15\%) being dependent on bathing or reporting urinary/fecal incontinence. The fTRST score was found to be $\geq 2$ in $51.3 \%$ of the patients $(40 / 78)$. The most frequent reported issues were polypharmacy (43/78 patients; $55.1 \%)$ and walking disability (31/78; 39.7\%). One 


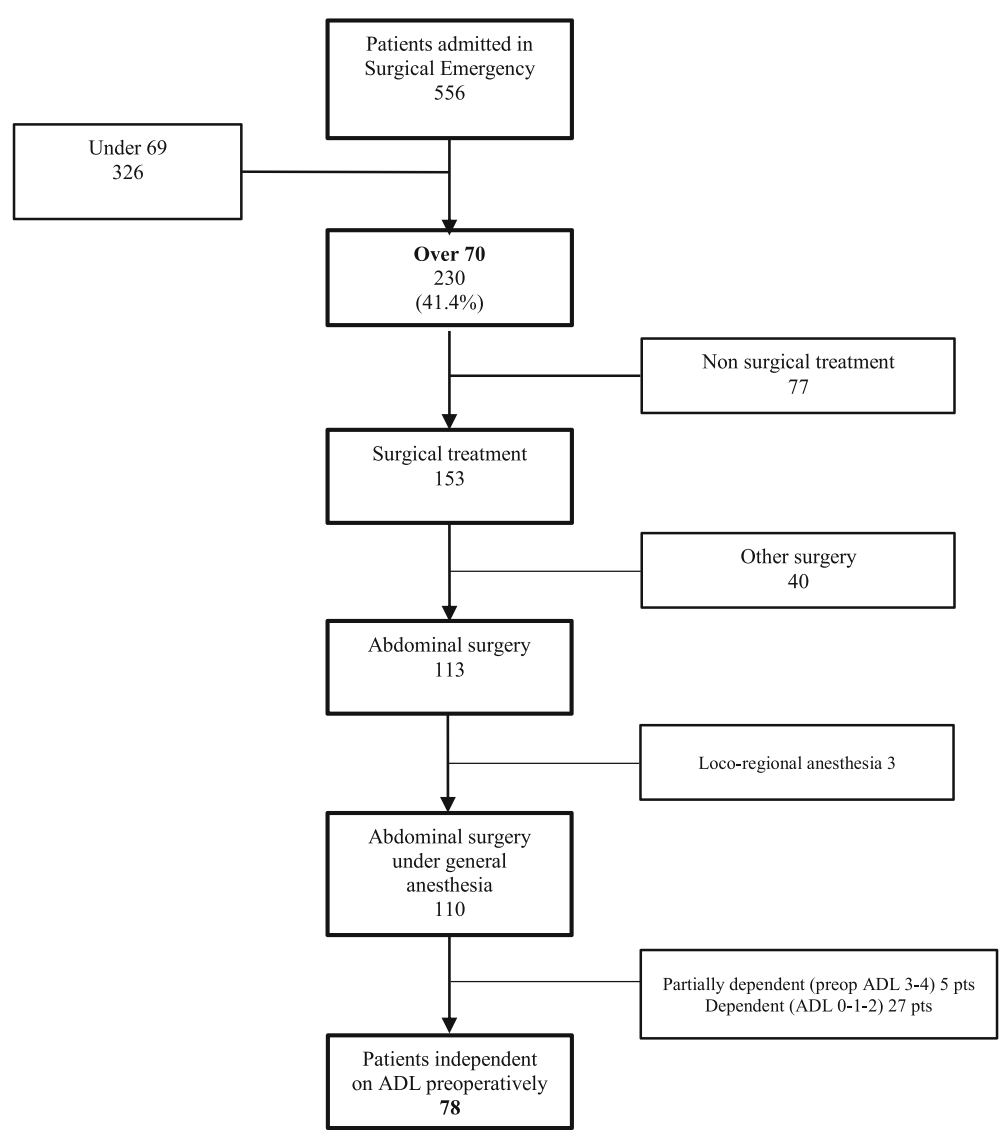

Fig. 1 Population diagram

in four independent patients lived alone with no available caregiver. The most commonly represented ASA score was ASA $3(68 \%)$. Almost half of the study population (38/78) presented a high comorbidity rate with elevated $\mathrm{CACI}$ score $(\mathrm{CACI} \geq 6)$.

\section{Surgical intervention}

The details of the surgical procedures are shown in Table 3. Most of the patients underwent operations for large or small bowel diseases (51/78 patients), with twenty patients found to be affected by colorectal cancer (Table 3). A major intervention was performed in 41 cases $(52.6 \%)$ including a colonic, small bowel, or gastric resection. Five cases of cholecystectomy were considered as major procedure, in four cases because of the presence of a diffuse biliary peritonitis requiring open approach or conversion to open procedure, and in one case because of the presence of duct obstruction requiring an endoscopic retrograde cholangiopancreatography during the same procedure. Thirty-eight procedures were performed of intermediate and minor surgery (cholecystectomies, appendectomies, hernia repair, adhesiolysis, stoma creation without bowel resection). Surgery was performed laparoscopically in $22 \%$ of the cases.

\section{Mortality}

The postoperative 30 -day mortality rate was $12.8 \%$ (10/ 78 ) and occurred shortly after the intervention in 9 cases out of 10. The most frequent cause of death was septic shock. Seven patients had ASA score $\geq 4$ (7/10). Eight deaths were observed after a major procedure. The overall 90 -day mortality rate was $15.4 \%$ (12/78). The two deaths that occurred between 31 and 90 days were secondary to progression of an end-stage neoplasia.

\section{Complications}

Twenty patients $(25.6 \%)$ experienced an uneventful postoperative course. Twenty-six patients (26/78, 33.3\%) were transferred on ICU after the surgical procedure for postoperative monitoring.

The overall postoperative 30-day complication rate was $70.6 \%(48 / 68)$ (Table 4). Fifty percent of patients presented with non-surgical/medical complications. CD1 complications accounted for $50 \%$ (24/48) of all complications, which were mostly wound-related problems and prolonged ileus. While the Clavien-Dindo Classification only provides information about the worst complication occurring, in our study, 24 patients with a CD1-4 developed multiple complications. Among patients with 
Table 2 Demographic data

\begin{tabular}{|c|c|c|c|}
\hline \multirow{18}{*}{$\begin{array}{l}\text { Demographic } \\
\text { data }\end{array}$} & \multirow[t]{2}{*}{ Age } & Median (range) & $78(70-96)$ \\
\hline & & Over 85 n (\%) & $21(27 \%)$ \\
\hline & \multirow[t]{2}{*}{ Gender } & Male & 34 \\
\hline & & Female & $44(56.4 \%)$ \\
\hline & \multirow[t]{5}{*}{ ASA } & । & 0 \\
\hline & & $\|$ & $12(15.4 \%)$ \\
\hline & & III & $53(67.9 \%)$ \\
\hline & & IV & $12(15.4 \%)$ \\
\hline & & V & $1(1.3 \%)$ \\
\hline & \multirow[t]{2}{*}{$\mathrm{CACl}$} & Average (range) & $5(3-14)$ \\
\hline & & Score $\geq 6(\%)$ & $38(48.7 \%)$ \\
\hline & fTRST & Score $\geq 2(\%)$ & $40(51.3 \%)$ \\
\hline & \multirow[t]{4}{*}{ Walking capacity } & Any problem & $54(69.2 \%)$ \\
\hline & & With a cane & $18(23.1 \%)$ \\
\hline & & With two canes & $2(2.6 \%)$ \\
\hline & & With a walker & $4(5.1 \%)$ \\
\hline & \multirow[t]{2}{*}{ ADL } & ADL 5 (\%) & $12(15.4 \%)$ \\
\hline & & ADL 6 (\%) & $66(84.6 \%)$ \\
\hline \multirow[t]{11}{*}{ Diagnosis } & \multirow[t]{5}{*}{ Bowel } & $\begin{array}{l}\text { Obstruction } \\
\text { (cancer) }\end{array}$ & $32(11)$ \\
\hline & & $\begin{array}{l}\text { Perforation } \\
\text { (cancer) }\end{array}$ & $6(5)$ \\
\hline & & Diverticulitis & 5 \\
\hline & & Ischemia & 4 \\
\hline & & $\begin{array}{l}\text { Bleeding } \\
\text { (cancer) }\end{array}$ & $4(4)$ \\
\hline & Cholecystitis & $\begin{array}{l}n \text { (with } \\
\text { peritonitis) }\end{array}$ & $10(3)$ \\
\hline & $\begin{array}{l}\text { Gastric/duodenal } \\
\text { perforation }\end{array}$ & $n$ & 3 \\
\hline & Splenic injury & $n$ & 1 \\
\hline & Abdominal wall hernia & $n$ & 9 \\
\hline & Appendicitis & $n$ & 4 \\
\hline & TOTAL & $\boldsymbol{n}$ (cancer \%) & $78(25.6 \%)$ \\
\hline
\end{tabular}

multiple complications, a detailed morbidity analysis showed that postoperative delirium occurred in 7 patients, and congestive heart failure was observed in 9 cases.

\section{Length of stay}

The average postoperative length of stay (LOS) was 7 days in the emergency surgery unit and 10.26 days considering the entire hospital admission.

\section{Functional loss}

A severe degree of functional decline was observed in one out of four patients who were alive 30 days after surgery $(17 / 68,25 \%)$. The regression model (Table 5) showed that $\mathrm{fTRST} \geq 2, \mathrm{CACI} \geq 6$, and age $\geq 85$ years old had a significant relationship with the loss of independence in ADLs at 30 days. Analysis of the fTRST showed the most accuracy given the area under the curve (AUC) of 71.6 with a sensitivity of $76.5 \%$ and specificity of $66.7 \%$. Being $\geq 85$ years old was also a risk factor with a PPV (positive predictive value) of $60 \%$. When fTRST model was adjusted for age as continuous variable, the AUC increased to $80.57 \%$. A statistically significant odds ratio (OR) was obtained for fTRST, $\mathrm{CACI} \geq 6$, and age $\geq$ 85 (OR 6.5, 3.67, 8.44 respectively).

Between 31 and 90 days, no patient developed additional functional loss. However, in patients who had functional decline at 30 days, only $3 / 17$ patients recovered by 90 days, and 2 patients died in this period.

The regression model showed a significant relation between 90-day functional loss for fTRST, CACI, and age over 84 years. The most important predictor was the fTRST with an AUC of 72. When fTRST was adjusted per age as continuous variable, the AUC increased again to 77.27 .

\section{Institutionalization}

The institutionalization rate was $7.6 \%(5 / 66)$. The median age was 77 years old (range 75-92). All the institutionalized patients had a fTRST $\geq 2$. All the patients discharged to a nursing home lived alone or had no caregiver able or available before surgery (a variable also reported in the fTRST). All institutionalized patients developed a surgical complication during the hospital stay; 3 patients had a minor complication (CD1-2) and 2 a major complication (CD 4a-respiratory insufficiency and abdominal sepsis).

\section{Discussion}

The number of older adults admitted every year to surgical departments for an acute condition is rising, and 40$50 \%$ of all emergency surgical operations are performed in patients over 65 years of age [21, 22]. In our study, 41.4\% of patients admitted to our emergency surgery unit were 70 years old and older. Among this heterogeneous population, frailty is considered the most important risk factor for postoperative adverse events, prolonged length of stay, and functional and cognitive capacity decline $[4,9,23]$. Decision making in an emergency condition can be challenging, and frailty assessment becomes paramount in order to help clinicians identify patients at higher risk for poor outcomes and to discuss strategies and expectations with the patient and relatives. In the emergency setting, a comprehensive frailty assessment cannot usually be performed, not only because it would be too time consuming or because a geriatrician is not always available, but also because ongoing acute conditions incumber a reliable evaluation of the patient. In addition, some domains of 
Table 3 Surgical intervention

\begin{tabular}{|c|c|c|c|}
\hline Intervention & & Procedures & Major procedures \\
\hline Colonic resection & $\begin{array}{l}\text { Right 6, left 5, partial 2, ileocecal 1, sigmoid 1, } \\
\text { Hartmann 6, total colectomy } 2\end{array}$ & 23 (4 lap) & 23 \\
\hline Small bowel resection & & 8 & 8 \\
\hline Small bowel surgery (without resection) & adhesiolysis 16 , stoma 2 & 18 (2 lap) & / \\
\hline Cholecystectomy & & 10 (6 lap) & 5 \\
\hline Appendectomy & & 4 (4 lap) & / \\
\hline Hernia repair & & 9 & / \\
\hline Peptic ulcer repair & & 3 (1 lap) & 2 \\
\hline Gastric resection & & 1 & 1 \\
\hline Splenectomy & & 1 & 1 \\
\hline Abdominal lavage & & 1 (1 lap) & / \\
\hline Total & & 78 (17 lap-21.8\%) & $40(51.3 \%)$ \\
\hline
\end{tabular}

Lap= laparoscopic

Table 4 Postoperative complications according to Clavien-Dindo Classification

\begin{tabular}{|c|c|c|c|c|c|c|c|}
\hline & CD1 & CD2 & CD3a & CD3b & CD4a & CD4b & CD5 \\
\hline \multicolumn{8}{|l|}{ 30-day complication } \\
\hline Wound problem & 9 & & & & & & \\
\hline Delirium & 3 & & & & & & \\
\hline lleus & 4 & 2 & & & & & \\
\hline Infection (pneumonia, urinary inf, abdominal sepsis) & & 10 & & & 2 & 1 & 4 \\
\hline Biliary fistula & & 1 & & & & & \\
\hline Pulmonary embolism & & & & & 1 & & \\
\hline Atrial fibrillation & & 2 & & & & & \\
\hline Blood transfusion & & 2 & & & & & \\
\hline Urinary retention & 3 & & & & & & \\
\hline Congestive heart failure & 3 & & & & & & \\
\hline Heart failure & & & & & 1 & & 2 \\
\hline Hemoperitoneum & & & & 1 & & & \\
\hline Respiratory failure & & & & & 2 & & 2 \\
\hline Kidney failure & 1 & & & & & & \\
\hline MOF (multiple organ failure) & & & & & & & 1 \\
\hline Pressure ulcers & 1 & & & & & & \\
\hline Complications secondary to progression of an end stage neoplasia & & & & & & & 1 \\
\hline Total & 24 & 17 & 0 & 1 & 5 & 1 & 10 \\
\hline \multicolumn{8}{|l|}{ 90-day complication } \\
\hline Stoma problem & 1 & & & & & & \\
\hline TPN & & 1 & & & & & \\
\hline Respiratory failure (ARDS) & & & & & 1 & & \\
\hline Complications secondary to progression of an end stage neoplasia & & & & & & & 2 \\
\hline Pressure ulcers & 1 & & & & & & \\
\hline Total & 2 & 1 & 0 & 0 & 1 & 0 & 2 \\
\hline
\end{tabular}




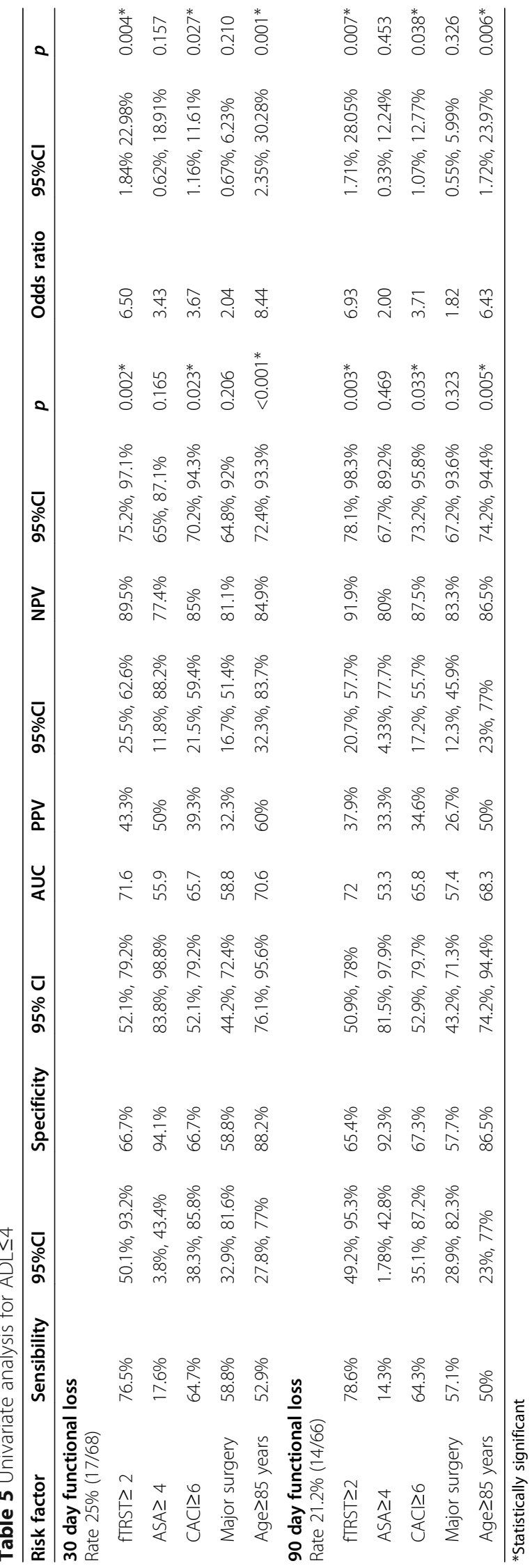


the frailty assessment such as walking capacity or mental status evaluation can be altered as a result of the acute condition. A reliable screening tool, feasible before emergency surgery, should have well-defined characteristics: (1) short and fast to perform, (2) requires limited blood tests as many are not available in the emergency setting (i.e., serum albumin level, interleukin), and (3) requires minimal patient collaboration/performance.

In the literature there are few studies on the application of frailty screening tools in the emergency setting. Among those, Joseph et al. [23] applied the modified 50variable Rockwood Preadmission Frailty Index (50-RPFI) among a geriatric surgical emergency population. This screening tool consists of 50 items and investigates many domains as comorbidities, ADL, IADL, and psychological, functional, and nutritional status, and requires specific blood test. A group of researchers from the University of Arizona validated in two publications [24, 25] a shorter form of this extensive questionnaire selecting the 15 variables more predictive of morbidity and mortality after emergency surgery, named emergency general surgery-specific frailty index (EGSFI). The EGSFI is easier to perform than the 50-RPFI and demonstrates a correlation with complications, mortality, adverse discharge disposition, and 30-day readmission rates [25].

Another screening tool applied in the emergency setting is the 7-point Clinical Frailty Scale (CFS) [26]. It is a simple infographic tool that classifies the patient in seven categories based on a clinician evaluation: very fit, well, well with treated comorbid disease, apparently vulnerable, mildly frail, moderate frail, severely frail. It was applied in different studies in trauma ad emergency surgery [9, 27, 28]. This screening tool seems reliable; however, this classification requires a comprehensive evaluation of comorbidities and several physical and psychological aspects. This clinical judgment requires a thorough knowledge of patient's past medical history and could be time consuming.

We previously described our experience using the fTRST as frailty screening tool in a population of older patients undergoing urgent abdominal surgery showing that it could accurately predict postoperative morbidity and mortality in this population [10].

In the present study, we investigated the role of the fTRST in predicting loss of independence, as functional recovery and maintenance of the preoperative functional capacity are often the most important outcomes to geriatric patients. Limited previous experiences have been reported in this field. Tan et al. [29] observed a loss of independence 1 year after surgery in $6.9 \%$ of the cohort and conclude that frailty (assessed with Modified Fried's frailty Criteria or with Modified Frailty Index-11) was the stronger predictor of functional decline. Unfortunately, the authors also revealed a major limitation in the application of the Modified Fried's frailty Criteria as frailty screening tool that they use as it requires to be sufficiently fit to complete the questionnaires, to perform the handgrip tests, and to walk a 15 - $\mathrm{ft}$ length twice. This overly complex tool is not always feasible in the emergency setting.

In our population, a postoperative functional decline was significantly predicted in patients age more than 85 years, presence of multiple comorbidities $(\mathrm{CACI} \geq 6)$, and if fTRST score was $\geq 2$ both at 30 and 90 days after surgery. The most accurate predictor according to the AUC in our study was found to be fTRST $\geq 2$.

Loss of independence has been established to be one of the most important outcomes among aged people because it impacts on patient quality of life, risk of institutionalization, caregiver/family and community burden, and health system financial costs [30, 31]. Functional loss in the geriatric population is often more feared than death; more than $70 \%$ of older people would not choose a treatment that causes severe functional disability, even if survival was assured [6] because independence and quality of life are strictly linked.

In our series, after an urgent surgical operation, the inability to perform ADLs among preoperatively independent senior adults was observed in $25 \%$ of patients 30 days after surgery. At the 3-month follow-up, we expected a functional recovery. Unfortunately, among the 17 patients who lost independence at 30 days, only 3 patients recovered based on their ADL scores. At the 90day mark, 2 patients died, 5 were permanently institutionalized, and 7 maintained the acquired decline in the ADL. Among functionally declined patients, at least one postoperative complication occurred within 30 days from surgery in every case. In $88.2 \%$ of cases, multiple complications were observed.

In the emergency setting where prehabilitation/preoperative optimization are not possible, the primary strategy to avoid functional decline is a prevention/early management of postoperative complications [5, 32] while adopting the main principles of the enhanced recovery protocols [33]. In our study, 50\% of all complications were non-surgical. Many patients reported multiple complications, the most frequent being delirium [7] and congestive heart failure [9]. To avoid loss of independence, a careful management of the postoperative course could help in reducing or mitigating medical complications and potentially impacting early and long-term outcomes. Preventive measures and perhaps establishing a geriatric comanagement program have been shown to be effective in managing postoperative delirium, cardiological complications, malnutrition, and promoting physical activity [34]. Shahrokni et al. [35] retrospectively evaluated the effects of geriatricians comanaging a cohort of 1020 patients who underwent elective cancer surgery compared to 872 similar patients who were treated without geriatric 
comanagement (standard surgical care). The adjusted probability of death within 90 days was less than half in the geriatric comanagement group (4.3\% versus $8.9 \%$, 95\% CI $2.3-6.9 \%, p<0.001)$. Although there were similar complication rates between the two groups, the geriatric comanagement group had greater utilization of supportive care services (e.g., physical therapy, nutrition services) and earlier recognition of geriatric-specific complications which likely contributed to the decreased mortality rate in this group.

Finally, for older patients undergoing surgery, it is of primary importance to identify patient's social support system (living situation, caregiver able or available) in order to optimize the discharge plan in advance and reduce prolonged hospitalization [34]. In our series, a discharge to a nursing-home facility occurred in $7.6 \%$ (5/ 66) of patients; all of them had a fTRST score $\geq 2$.

The main limitations of this study are the small sample size and single-center design. Heterogeneity of the surgical procedures could also represent a limitation; however, once more, we would like to highlight that in the emergency setting functional reserve (and consequently its preoperative evaluation) has a greater role compared to the surgical procedure itself. The follow-up was also limited to 3 months, although the decision to close the monitoring at that time was an attempt to reduce the chance that other causes, independent from the surgery, could influence the patient functional status.

\section{Conclusion}

Among functionally independent older patients, a feared consequence of a surgical intervention is loss of autonomy, becoming a burden for family/caregivers, or becoming institutionalized. Above all in the emergency setting, detection of frailty and careful prevention of postoperative complications are most effective in avoiding functional decline. fTRST is brief and easy to perform and allows identification of vulnerable and frail senior adults to effectively predict functional decline among preoperatively independent patients.

\section{Abbreviations}

ADL: Activities of daily living; fTRST: Flemish version of the Triage Risk Screening Tool; ASA: American Society of Anesthesiology Score; CACl: Charlson Comorbidity Age Adjusted; ESU: Emergency surgery unit; CD: Clavien-Dindo classification; ROC: Receiver operating characteristic curve; AUC: Area under the curve; OR: Odds ratio; AIC: Akaike information criterion; BIC: Bayesian information criterion; 50-RPFI: 50-variable Rockwood Preadmission Frailty Index; IADL: Instrumental activities of daily living; Cl: Confidence interval

\section{Acknowledgements}

Not applicable

\section{Related paper presentations}

This article is an original work, has not been published before, and is not being considered for publication elsewhere in its final form, in either printed or electronic media.

\section{Authors' contributions}

Study design: Zattoni, Montroni, Saur. Data collection: Zattoni, Garutti, Bacchi Reggiani. Analysis and interpretation of data: Bacchi Reggiani (statistical analysis), Montroni, Ugolini. Drafting of manuscript: Zattoni, Montroni, Saur, Ghignone, Taffurelli. Critical revision: Ugolini, Saur. The authors read and approved the final manuscript.

\section{Funding}

No funding was received.

\section{Availability of data and materials}

The dataset generated and analyzed during the current study is available from the corresponding author on reasonable request.

\section{Declarations}

Ethics approval and consent to participate

The study was conducted under the Institutional Review Board (214/2016/O/ OssN). Informed consent was obtained from every patient, and a health care proxy was used in cases of dementia or altered mental status. All participants gave their consent to the publication.

\section{Consent for publication}

Each author agrees to the publication of the present study.

\section{Competing interests}

The authors declare that they have competing interests.

\section{Author details}

${ }^{1}$ Department of General Surgery, Policlinico S. Orsola-Malpighi, 40138 Bologna, Italy. ${ }^{2}$ Department of General Surgery, Ospedale per gli Infermi, Viale Stadone, 9, -48018 Faenza, Italy. ${ }^{3}$ Department of Surgery, Division of Colon and Rectal Surgery, University of Pennsylvania, 800 Walnut Street 20th floor, Philadelphia, PA, USA. ${ }^{4}$ Department of Geriatrics, Policlinico S. Orsola-Malpighi, 40138 Bologna, Italy. ${ }^{5}$ Department of Cardiology-Statistics division, Policlinico S. Orsola-Malpighi, 40138 Bologna, Italy.

Received: 1 February 2021 Accepted: 26 February 2021

Published online: 18 March 2021

\section{References}

1. Ukkonen $\mathrm{M}$, Jämsen $\mathrm{E}$, Zeitlin R, Pauniaho S-L. Emergency department visits in older patients: a population-based survey. BMC Emerg Med. 2019;19(1):20

2. Desserud KF, Veen T, Søreide K. Emergency general surgery in the geriatric patient. Br J Surg. 2016;103(2):e52-61.

3. Bergenfelz A, Søreide K. Improving outcomes in emergency surgery. Br J Surg. 2014;101(1):e1-2

4. Mclsaac DI, Moloo H, Bryson GL, van Walraven C. The association of frailty with outcomes and resource use after emergency general surgery: a population-based cohort study. Anesth Analg. 2017;124(5):1653-61.

5. Torrance ADW, Powell SL, Griffiths EA. Emergency surgery in the elderly: challenges and solutions. Open Access Emerg Med. 2015;7:55-68.

6. Fried TR, Bradley EH, Towle VR, Allore H. Understanding the treatment preferences of seriously ill patients. N Engl J Med. 2002;346(14):1061-6.

7. Ellis G, Marshall T, Ritchie C. Comprehensive geriatric assessment in the emergency department; 2014. p. 2033-43.

8. Farhat JS, Velanovich V, Falvo AJ, Horst HM, Swartz A, Patton JHJ, et al. Are the frail destined to fail? Frailty index as predictor of surgical morbidity and mortality in the elderly. J Trauma Acute Care Surg. 2012;72(6):1521-6.

9. Goeteyn J, Evans LA, De Cleyn S, Fauconnier S, Hewitt J, Ceelen W, et al. Frailty as a predictor of mortality in the elderly emergency general surgery patient. Acta Chir Belg. 2017;0(0):1-6. https://doi.org/10.1080/00015458.201 7.1337339 .

10. Zattoni D, Montroni I, Saur NM, Garutti A, Bacchi Reggiani ML, Galetti C, et al. A simple screening tool to predict outcomes in older adults undergoing emergency general surgery. J Am Geriatr Soc. 2019;67(2): 309-16.

11. Skala K, Gervaz P, Buchs N, Inan I, Secic M, Mugnier-Konrad B, et al. Risk factors for mortality-morbidity after emergency-urgent colorectal surgery. Int J Color Dis. 2009;24(3):311-6. 
12. St-Louis E, lqbal S, Feldman LS, Sudarshan M, Deckelbaum DL, Razek TS, et al. Using the age-adjusted Charlson comorbidity index to predict outcomes in emergency general surgery. J Trauma Acute Care Surg. 2015; 78(2):318-23.

13. Laor A, Tal S, Guller V, Zbar AP, Mavor E. The Charlson Comorbidity Index $(\mathrm{CCl})$ as a mortality predictor after surgery in elderly patients. Am Surg. 2016;82(1):22-7.

14. Audisio RA, Pope D, Ramesh HSJ, Gennari R, van Leeuwen BL, West C, et al. Shall we operate? Preoperative assessment in elderly cancer patients (PACE) can help. A SIOG surgical task force prospective study. Crit Rev Oncol Hematol. 2008;65(2):156-63.

15. Merani S, Payne J, Padwal RS, Hudson D, Widder SL, Khadaroo RG. Predictors of in-hospital mortality and complications in very elderly patients undergoing emergency surgery. World J Emerg Surg. 2014;9:43.

16. Al-Temimi MH, Griffee M, Enniss TM, Preston R, Vargo D, Overton S, et al. When is death inevitable after emergency laparotomy? Analysis of the American College of Surgeons National Surgical Quality Improvement Program database. J Am Coll Surg. 2012;215(4):503-11.

17. Kenis C, Geeraerts A, Braes T, Milisen K, Flamaing J, Wildiers H. 19 The Flemish version of the Triage Risk Screening Tool (TRST): a multidimensional short screening tool for the assessment of elderly patients. Crit Rev Oncol Hematol. 2006;60:\$31.

18. Oh NH, Kim KJ. Outcomes and risk factors affecting mortality in patients who underwent colorectal emergency surgery. Ann Coloproctol. 2016;32(4): $133-8$.

19. Lees MC, Merani S, Tauh K, Khadaroo RG. Perioperative factors predicting poor outcome in elderly patients following emergency general surgery: a multivariate regression analysis. Can J Surg. 2015;58(5):312-7.

20. Duron J-J, Duron E, Dugue T, Pujol J, Muscari F, Collet D, et al. Risk factors for mortality in major digestive surgery in the elderly: a multicenter prospective study. Ann Surg. 2011;254(2):375-82

21. Wittenberg R, Sharpin L, McCormick B, Hurst J. Understanding emergency hospital admissions of older people. Centre for Health Service Economics \& Organisation (CHSEO). Report No. 6; 2014

22. Makary MA, Segev DL, Pronovost PJ, Syin D, Bandeen-Roche K, Patel P, et al. Frailty as a predictor of surgical outcomes in older patients. J Am Coll Surg. 2010;210(6):901-8.

23. Joseph B, Zangbar B, Pandit V, Fain M, Mohler MJ, Kulvatunyou N, et al. Emergency general surgery in the elderly: too old or too frail ? J Am Coll Surg. 2016;222(5):805-13. https://doi.org/10.1016/j.jamcollsurg.2016.01.063.

24. Jokar TO, Ibraheem K, Rhee P, Kulavatunyou N, Haider A, Phelan HA, et al. Emergency general surgery specific frailty index: a validation study. J Trauma Acute Care Surg. 2016;81(2):254-60.

25. Khan M, Jehan F, Zeeshan M, Kulvatunyou N, Fain MJ, Saljuqi AT, et al. ScienceDirect failure to rescue after emergency general surgery in geriatric patients: does frailty matter? J Surg Res. 2018;233:397-402. https://doi.org/1 0.1016/j.jss.2018.08.033

26. Rockwood K, Song X, MacKnight C, Bergman H, Hogan DB, McDowell I, et al. A global clinical measure of fitness and frailty in elderly people. CMAJ. 2005;173(5):489-95.

27. Eamer GJ, Clement F, Holroyd-Leduc J, Wagg A, Padwal R, Khadaroo RG. Frailty predicts increased costs in emergent general surgery patients: a prospective cohort cost analysis. Surgery. 2019;166(1):82-7.

28. Cheung A, Haas B, Ringer TJ, McFarlan A, Wong CL. Canadian Study of Health and Aging Clinical Frailty Scale: does it predict adverse outcomes among geriatric trauma patients? J Am Coll Surg. 2017;225(5):658-665.e3.

29. Tan HL, Theng S, Chia X, Nadkarni NV, Ang SY. Frailty and functional decline after emergency abdominal surgery in the elderly: a prospective cohort study; 2019. p. 1-7.

30. Becher RD, Murphy TE, Gahbauer EA, Leo-Summers L, Stabenau HF, Gill TM. Factors associated with functional recovery among older survivors of major surgery. Ann Surg. 2020;272(1):92-8.

31. Scott JW, Olufajo OA, Brat GA, Rose JA, Zogg CK, Haider AH, et al. Use of national burden to define operative emergency general surgery. JAMA Surg. 2016;151(6):e160480

32. Zattoni D, Christoforidis D. How best to palliate and treat emergency conditions in geriatric patients with colorectal cancer. Eur J Surg Oncol. 2020;46(3):369-78.

33. Lohsiriwat $V$, Jitmungngan $R$. Enhanced recovery after surgery in emergency colorectal surgery: review of literature and current practices. World J Gastrointest Surg. 2019;11:41-52.
34. Berian JR, Mohanty S, Ko CY, Rosenthal RA, Robinson TN. Association of loss of independence with readmission and death after discharge in older patients after surgical procedures. JAMA Surg. 2016;151(9):e161689.

35. Shahrokni A, Tin AL, Sarraf S, Alexander K, Sun S, Kim SJ, et al. Association of geriatric comanagement and 90-day postoperative mortality among patients aged 75 years and older with cancer; 2020. p. 1-11.

\section{Publisher's Note}

Springer Nature remains neutral with regard to jurisdictional claims in published maps and institutional affiliations.
Ready to submit your research? Choose BMC and benefit from:

- fast, convenient online submission

- thorough peer review by experienced researchers in your field

- rapid publication on acceptance

- support for research data, including large and complex data types

- gold Open Access which fosters wider collaboration and increased citations

- maximum visibility for your research: over $100 \mathrm{M}$ website views per year

At BMC, research is always in progress.

Learn more biomedcentral.com/submissions 